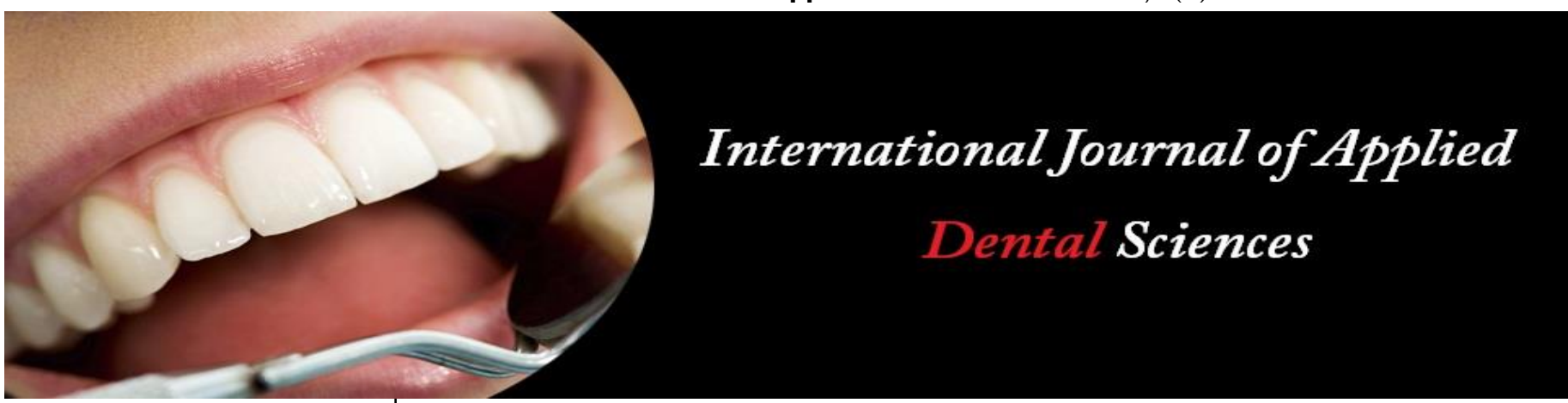

ISSN Print: 2394-7489

ISSN Online: 2394-7497

IJADS 2018; 4(4): 332-335

(C) 2018 IJADS

www.oraljournal.com

Received: 21-08-2018

Accepted: 23-09-2018

\section{Parkavi A}

Department of Periodontics,

Nair Hospital Dental College,

Maharashtra University of

Health Sciences, Mumbai,

Maharashtra, India

Mala Dixit Baburaj

Department of Periodontics,

Nair Hospital Dental College,

Maharashtra University of

Health Sciences, Mumbai,

Maharashtra, India

\section{Correspondence}

Parkavi A

Department of Periodontics,

Nair Hospital Dental College,

Maharashtra University of

Health Sciences, Mumbai,

Maharashtra, India

\section{Oral mucocoele: A case report}

\section{Parkavi A and Mala Dixit Baburaj}

\section{Abstract}

The mucocele is one of the most common benign soft tissue masses that occur in the oral cavity. Diagnosis is mostly based on clinical findings. The common location of the extravasation mucocele is the lower lip and the treatment of choice is surgical removal. This paper gives an insight into the phenomenon and a case report has been presented.

Keywords: Mucocoele, mucus retention cyst, mucus extravasation cyst, lip swelling

\section{Introduction}

The term mucocoele is derived from the Latin word Mouco: meaning mucus and Coele: meaning cavity. They are basically "mucus filled cavities" usually present in the oral cavity, lacrimal sac, and paranasal sinuses.

By definition, they are not true cysts. The incidence is high, in the order of 2.5 lesions per 1000 individuals ${ }^{[1]}$. Indeed, mucsoceles are the most common minor salivary gland disorder, and represent the second most frequent benign soft tissue tumor of the oral cavity, following irritative fibromas ${ }^{[2]}$.

\subsection{Types}

Clinically there are two types, extravasation and retention type. Extravasation type is due to the leaking of fluid from the damaged salivary gland ducts and acini into the surrounding soft tissues, mostly seen in minor salivary glands. Retention type is due to the blockage of salivary gland duct, seen commonly in major salivary gland ducts ${ }^{[3,4]}$. Clinically there is no difference between both.

Mucoceles present as bluish, soft, transparent cystic swelling that frequently resolve spontaneously. Blue colour is due to vascular congestion, cyanosis of the tissue above, and accumulation of fluid below. However, colour may vary depending on the size of the lesion, proximity to the surface, and elasticity of overlying tissue ${ }^{[5]}$. When this mucocele is located in floor of the mouth it appears as the underbelly of a frog, hence called as ranula ${ }^{[6]}$.

\subsection{Etiopathogenesis}

The two main etiological factors are:

- Trauma

- Obstruction of salivary gland duct

Mainly physical trauma can cause spillage of salivary secretion into surrounding submucosal tissue. Later inflammation may become obvious due to stagnate mucous ${ }^{[3,4]}$. Habit of lip biting and tongue thrusting are also one of the aggravating factors ${ }^{[3,7]}$.

The extravasation type will undergo three evolutionary phases ${ }^{[3,8]}$. In first phase, there will be spillage of mucous from salivary duct in to the surrounding tissue in which some leucocytes and histiocytes are seen. In second phase called resorption phase, granulomas will appear due to the presence of histiocytes, macrophages and giant multinucleated cells associated with a foreign body reaction. Later in third phase, there will be a formation of pseudocapsule without epithelium around the mucosa due to connective cells. The retention type of mucocele is commonly seen in major salivary glands due to the dilation of duct caused by a sialolith or dense mucosa ${ }^{[3,8]}$ 
Although minor salivary glands are found in most parts of the oral cavity except the gingiva, mucoceles occur most commonly in the lower lip, probably due to the higher incidence of mechanical trauma in this region ${ }^{[9,10]}$. Children and young adults are most commonly affected, although these lesions can occur at any age. Both sexes are equally affected [9]

Generally, small and superficial mucoceles do not require treatment because they often heal after spontaneous rupture [9]. Treatment options include surgical excision, marsupialization, micro marsupialization, cryosurgery, laser vaporization, and laser excision. However, in most cases, the treatment of choice is excision. The lesions can be completely excised, including the associated salivary gland tissue as well as any marginal glands, before primary closure, reducing the incidence of recurrence ${ }^{[9]}$.

\section{Case report}

\subsection{Clinical examination}

A 23 years old male patient had reported to the Department of Periodontics, Nair Hospital Dental College, Mumbai, with the chief complaint of swelling present in the lower lip since the last 3 weeks.

The patient was apparently all right three weeks back when he noticed a small swelling in the lower lip initially, which then gradually increased to the present size. He gave history of lip biting habit. There was no pain associated with the swelling. His past medical, dental and drug history were not significant. There were no contributory family history.

On extra oral examination, the face appeared bilaterally symmetrical, with competent lips and the lymph nodes were not palpable. On oral examination a solitary round sessile palpable fluctuant non-tender swelling with no increase in temperature was present on the left lower labial mucosa with respect to 32 and 33 . The swelling had a reddish blue hue compared to the adjacent mucosa. There was no difficulty in speaking or chewing. There was no evidence of calcification or retained foreign body present. No other oral anomalies were detected. Based on the history and clinical features a provisional diagnosis of mucocoele with respect to 32,33 was given.
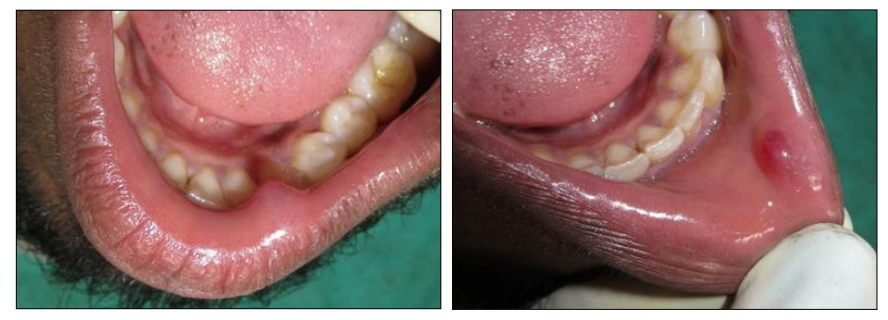

Fig 1 A, B: Pre-operative presentation of swelling

\subsection{Investigations}

Routine hematological examinations including hemogram, bleeding and clotting time were found to be within normal physiological limits.

\subsection{Differential diagnosis}

The differential diagnosis consisted of fibroma, lipoma soft tissue abscess, oral hemangioma, oral lymphangioma, benign and malignant salivary gland neoplasms, Cicatricial pemphigoid, and Bullous lichen planus.

Fibromas vary in consistency from soft to very firm. They are the most common intraoral soft-tissue lesion and are seen most frequently on the lips. Lipomas, neoplasms consisting of mature adipose tissue, are uncommon in the oral cavity, but can occur on the lips. Lipomas and tumours of minor salivary glands present no fluctuation while cysts, mucoceles, abscess, and hemangiomas show fluctuation. Vascular malformations such as hemangiomas and varices are usually blue in colour, blanch under digital pressure.

The appearance of mucoceles is pathognomonic and the following data are crucial: lesion location, history of trauma, rapid appearance, variations in size, bluish colour and the consistency. Based on history and clinical examination a provisional diagnosis of mucocele was made. Surgical excision of the lesion was planned using scalpel under local anesthesia.

\subsection{Treatment}

Phase I therapy consisted of a thorough scaling and root planing and oral hygiene instruction were given. The patient was demonstrated the proper brushing technique (Modified Bass Technique). Patient was adviced $0.2 \%$ chlorhexidine mouth-rinse $10 \mathrm{ml}, 1: 1$ dilution, twice daily for 15 days.

Phase II therapy consisted of surgical excision of the lesion under local anesthesia. The patient was explained about the procedure and informed consent was obtained. Local infiltrative anesthesia was applied around the lesion. The anesthetic was not infiltrated directly into the lesion to avoid compromising the biopsy. The lip was then everted with digital pressure to increase the lesion's prominence. First a semi-circular incision was made around the lesion to obtain a proper biopsy sample and to decrease the extent of mucosal tissue loss, decreases the incidence of formation of large fibrous scars, and helps to prevent spilling of the cystic content, which could be responsible for recurrence. Dissection was performed, separating the lesion and associated minor salivary gland. To reduce the chance of recurrence, lesion was removed down to the muscle layer, all the surrounding glandular acini were excised, and damage to the adjacent gland and duct were avoided while placing the suture. The surgical site was then thoroughly irrigated and then the wound margins were approximated and sutured with silk sutures. The specimen was sent for histopathologic examination.

Post-operative instructions were given and analgesics were prescribed. Patient was recalled after 1 week for the removal of sutures and a satisfactory wound healing was seen. No recurrence was seen after a follow up at 1 month, 3 months and 1 year.
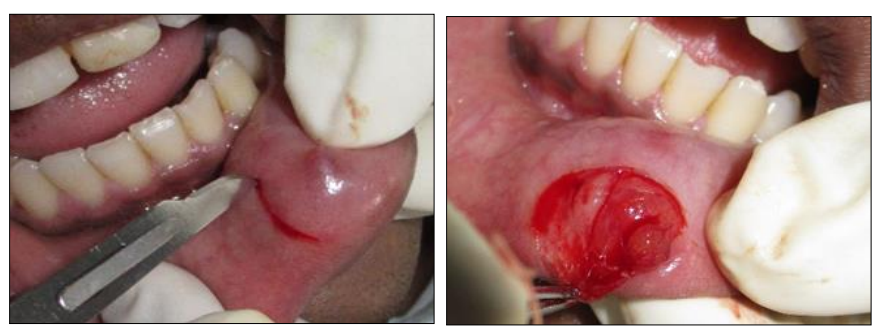

Fig 2A: Incision B: exposure of the swelling under local anesthesia
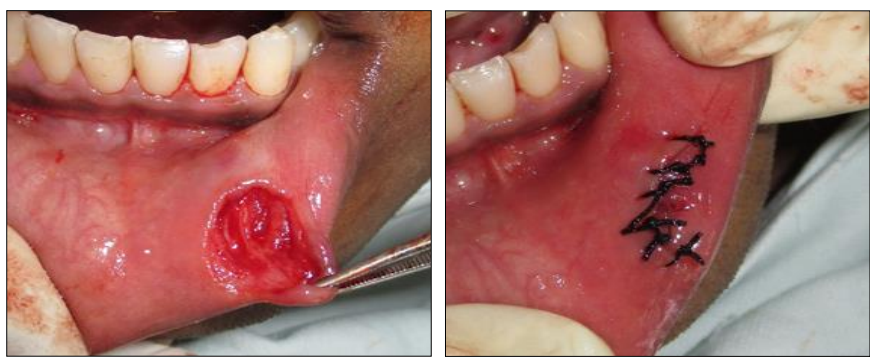

Fig 3 A: In Toto excision of the cyst, B: sutures placed 

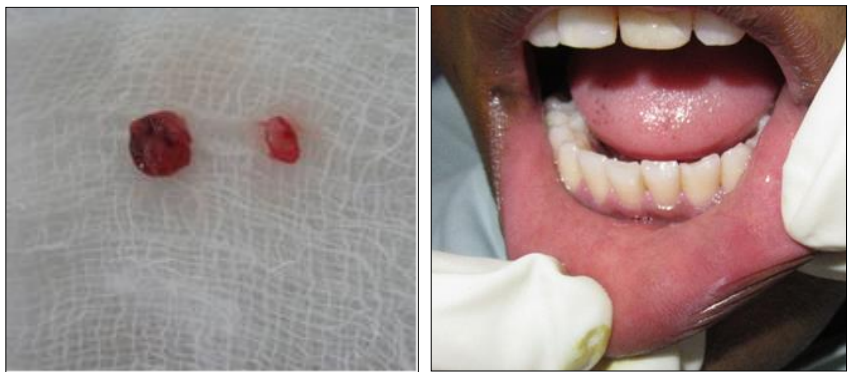

Fig 4 A: Excised mucocoele, B: 1 month post-operative presentation

\subsection{Histopathology}

The overall histopathological features were suggestive of mucous extravasation cyst confirming the diagnosis of mucocoele.
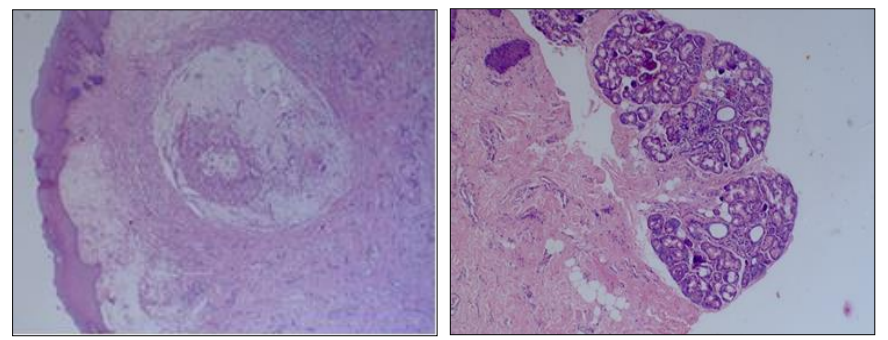

Fig 5: Histopathologic confirmation of mucous extravasation cyst

\section{Discussion}

The incidence of mucoceles in the general population is $0.4 \%$ to $0.8 \%$ with scant differences between males and females ${ }^{[1]}$. Lower lip is considered to be the most frequently affected location ( $40 \%$ to $80 \%$ of all cases), followed by the cheek mucosa and floor of the mouth ${ }^{[1,6]}$.

It is generally determined by a traumatic event that can cause the rupture of an excretory duct of salivary gland and usually appears as solitary fluctuant non-tender swelling in caninebicuspid area with a normal pink or bluish colour. In the present case, the patient gave a history of lip biting habit in the 32,33 area, which ultimately lead to mucocoele formation at the site due to the repeated trauma.

The history and the clinical features are pathognomic leading to the diagnosis of mucocoele. Fine needle aspiration demonstrates the mucus retention, histiocytes and inflammatory cells $[3,11]$. Chemical analysis shows high amylase and protein content. Radiographs are the contributing factors in cases of ranulas ${ }^{[3]}$. Localization of these lesions is done by Computed Tomography and Magnetic Resonance Imaging ${ }^{[3,7]}$.

Histopathologic examination of mucocele often reveals formation of well-circumscribed, cyst-like space surrounded by granulation tissue and the presence of mucino phages in the collapsed wall of granulation tissue ${ }^{[12,13]}$. The adjacent salivary gland tissue should also be present because mucocele should always be removed along with feeder glands/ducts which minimize recurrence of the lesion ${ }^{[12]}$. The present case was diagnosed as mucus extra vsation cyst histopathologically.

The primary objective in the treatment of mucocele is the complete resection of the lesion in order to prevent its recurrence. We have to ensure that both the affected and neighboring glands are removed along with the pathological tissue to avoid relapse. Several techniques have been proposed for the treatment of a mucocele, such as cryosurgery, micro marsupialization, marsupialization, surgical excision and laser ablation ${ }^{[1]}$.
The conventional method is the treatment by surgical approach. It does not require extensive equipment, has negligible cost, and can be performed by most trained dentists. It does require great precision, however, and detailed knowledge of the mucocele and the surrounding anatomy is must ${ }^{\text {[9] }}$. It also requires great control of the instrument, with accurate tactile awareness as there is a chance for rupturing the mucocele and leakage of its contents can cause soft tissue collapse with loss of anatomical references needed for resection. Hence, special care is mandatory to avoid damage to other glands or ducts while suturing because this can even lead to its recurrence.

In our case report we excised the lesion using scalpel. We didn't experience extensive bleeding and the healing was uneventful. We adopted this procedure mainly because it's easy and economical compared to laser ablation, cryosurgery and electro cautery.

Marsupialization has resulted in considerably higher recurrence rates [1]. Micro marsupialization has been suggested to have lower recurrence rates, although it is restricted to lesions with clinical characteristics that strongly suggested a diagnosis of mucocele, since histopathological examination is not possible ${ }^{[1,14]}$.

Cryosurgery has yielded satisfactory results with no recurrence. Reported postoperative symptoms, however, included marked edema and irritation, as well as a prolonged healing time ${ }^{[1,15]}$.

Vaporization with argon and $\mathrm{Nd}$ : YAG lasers has been described as a new technique for the treatment of mucoceles. Both lasers procedures presented satisfactory results with low recurrence rates and were well tolerated by the patients, whose discomfort was the main complaint reported ${ }^{[1,16]}$.

The main advantages of soft tissue laser applications are minimal intraoperative bleeding, swelling and postoperative pain and very less surgical time, scarring, and coagulation, without any need of suturing after excision because of natural wound dressing due to denatured proteins ${ }^{[12]}$.

\section{Conclusion}

Mucocele is the most common benign self-limiting condition. Trauma is the most common cause and therefore identification and treatment of the associated habits becomes essential. Majority of these lesions are seen in the lower lip which can be disturbing in function and un-esthetic to the patient. Simple surgical excision is the treatment of choice and when done with care, it is the best treatment that can relive the patient's anxiety and discomfort.

\section{References}

1. Navya LV, Sabari C, Seema G. Excision of Mucocele by Using Diode Laser: A Case Report, Journal of Scientific Dentistry, 2016, 6(2).

2. Baurmash H. The etiology of superficial oral mucoceles. J Oral Maxillofac Surg. 2002; 60:237-238.

3. Prasanna Kumar Rao, Shishir Ram Shetty, Laxmikanth Chatra, Prashanth Shenai. Oral Mucocele-A Mini Review, Dentistry 3:153. doi:10.4172/21611122.1000153

4. Boneu-Bonet F, Vidal-Homs E, Maizcurrana-Tornil A, González-Lagunas J. Submaxillary gland mucocele: presentation of a case. Med Oral Patol Oral Cir Bucal. 2005; 10:180-184

5. Dr. Rini Gangwal Badjatia, Dr. SourabhBadjatia, Dr. Vinaya Kumar Kulkarni, Dr. Divya S. Sharma, Oral Mucocele A Case Report, NJDSR. 2014; 2:1.

6. Baurmash HD. Mucoceles and ranulas. J Oral Maxillofac 
Surg. 2003; 61:369378.

7. Gupta B, Anegundi R, Sudha P, Gupta M. Mucocele Two Case Reports. J Oral Health Comm Dent. 2007; 1:56-58.

8. Ata-Ali J, Carrillo C, Bonet C, Balaguer J, Peñarrocha M, et al. Oral mucocele: review of the literature. J Clin Exp Dent. 2010; 2:e18-e21.

9. Parveen Reddy KM, et al. Mucocele on the lower lip treated by scalpel excision method-A Case Report, Journal of Applied Dental and Medical Sciences, 2015; 1(3).

10. Gonsalves WC, Chi AC, Neville BW. Common oral lesions: Part II. Masses and neoplasia. Am Fam Physician. 2007; 75:509-512.

11. López-Jornet P, Bermejo-Fenoll A. Point of Care: What is the most appropriate treatment for salivary mucoceles? Which is the best technique for this treatment? J Can Dent Assoc. 2015; 70:484-485.

12. Subramaniam Ramkumar, Lakshmi Ramkumar, Narasimhan Malathi, Ramalingam Suganya. Case Report - Excision of Mucocele Using Diode Laser in Lower Lip, Case Reports in Dentistry, 2016, Article ID 1746316

13. Lee E, Cho SH, Park CJ. Clinical and immunohistochemical characteristics of mucoceles, Annals of Dermatology. 2009; 21(4):345-351.

14. Delbem AC, Cunha RF, Vieira AE, Ribeiro LL. Treatment of mucus retention phenomena in children by the micro marsupialization technique: Case reports. Pediatr Dent. 2000; 22:155-8.

15. Neumann RA, Knobler RM. Treatment of oral mucous cysts with an argon laser. Arch Dermatol. 1990; 126:82930 .

16. Jinbu Y, Tsukinoki K, Kusama M, Watanabe Y. Recurrent multiple superficial mucocele on the palate: Histopathology an laser vaporization. Oral Surg Oral Med Ora Pathol Oral Radiol Endod. 2003; 95:193-7. 